\title{
DER TAUSCH UND DER TOD. HESIOd - MAUSS - BATAILle - BAUdRILlaRd - SOPHOKLES
}

\author{
ACHIM GEISENHANSLÜKE
}

\section{Prometheus und Pandora bei Hesiod}

In Hesiods Theogonie, einem der frühesten Texte der abendländischen Literaturgeschichte, wird die Grundlage der menschlichen Kultur an eine bestimmte Form des Tausches gebunden. Die Geschichte vom Kräftemessen zwischen Zeus und Prometheus beginnt mit einem Opfer:

Als nämlich die Götter und die sterblichen Menschen sich trennten zu Mekone, da legte er ein großes Rind, nachdem er es bereitwillig geteilt hatte, vor, den Sinn des Zeus zu täuschen. Der einen Partei nämlich stellte er das Fleisch und die fetten Eingeweide in einer Haut hin, sie verhüllend mit dem Magen des Stiers, der anderen aber stellte er die weißen Knochen des Rindes, in listiger Kunst geordnet, hin, sie verhüllend mit glänzendem Fett. ${ }^{1}$

Der Mythos von Prometheus, nicht zu trennen von der Lehre der drei Weltzeitalter, die Hesiod in den Tagen und Werken entfaltet, erzählt von der Trennung von Menschen und Göttern als einem agonal strukturierten Vergleich, den Prometheus stellvertretend für die Menschen mit Zeus führt. Das Mittel, das Prometheus zur Verfügung steht, ist die List, die von Hesiod, im Unterschied etwa zu Aischylos, äußerst ambivalent beurteilt wird. Der Begriff der metis, ${ }^{2}$ der in der griechischen Tradition Prometheus wie schon Odysseus zugeordnet wird, steht für ein trügerisches, bunt schillerndes Wesen, dessen Kunst darin besteht, Schein und Sein zu vertauschen: Unter der unansehnlichen Haut und dem Magen des Stiers verbergen sich die kostbaren Fleischteile, unter dem glänzenden Fett die

1 Hesiod: Theogonie, hg, übers. u. erl. v. Karl Albert, 3. Aufl., Sankt-Augustin, Richarz 1985, V. 535-541.

2 Zur Bedeutung des Begriffes vgl. Renate Zoepffel: »Die List bei den Griechen «, in: Harro von Senger (Hg.), Die List, Frankfurt/Main 1999, S. 111133, besonders S. $112 f$. 
unverzehrbaren Knochen, die fortan als Opfer an die Götter verbrannt werden. Das Opfer des Prometheus vollzieht einen Tausch, der den Menschen zwar das Überleben sichert, sie zugleich aber in einen unauflösbaren Konflikt mit den Göttern stellt. Auf den Betrug des Prometheus antwortet Zeus daher mit einer Gegengabe, die die vielbeschworene Kongruenz von gift und Geschenk erfüllt. Den Menschen, denen Prometheus mit einer zweiten List das Feuer als Grundlage aller Technik gebracht hatte, schickt er die Pandora als, wie es bündig heißt, ein »schönes Übel (kalòn kakòn) «, ${ }^{3}$ als eine ebenso glänzende wie täuschende Gabe, die beständig zur Verschwendung anregt und die hart erarbeiteten Vorräte der Männer aufbraucht.

Hesiods Zusammenführung des Prometheus- und des Pandoramythos wirft über ihren unbestreitbar misogynen Aspekt hinaus einen grundsätzlich skeptischen Blick auf die menschliche Existenz. Die Trennung zwischen Menschen und Göttern stellt er als einen Betrug dar, der ein unaufhebbares Missverhältnis herstellt, das zwar zum einen als Ursprungsgeschichte aller kultureller Techniken begriffen werden kann, den Menschen jedoch zugleich mit Problemen konfrontiert, denen er nicht gewachsen ist. »Vordem nämlich lebten die Stämme der Menschen auf Erden fern von Übeln, elender Mühsal und quälenden Leiden, die Menschen den Tod bringen [...]. Das Weib aber hob mit den Händen den mächtigen Deckel vom Faß, ließ alles heraus und schuf der Menschheit leidvolle Schmerzen. « ${ }^{4}$ Was die Verschwendungssucht, die Hesiod der Frau unterstellt, den Menschen bringt, sind Krankheit und Schmerzen, letztlich der Tod. Die ambivalente Darstellung der Hoffnung in den Werken und Tagen, die als einziges Übel im von Pandora geöffneten Fass verbleibt, verweist auf die Endlichkeit der menschlichen Existenz als einer Grenze, die zu überschreiten auch der von Prometheus bereitgestellten Technik nicht möglich ist. Das einzige, was die Hoffnung vermag, ist, den Blick von dem jederzeit drohenden Ende des Lebens zu wenden, dem Menschen das Wissen des Todes zu nehmen, um ihn dadurch unwissend und doch dem Tod überantwortet für kurze Zeit am Leben zu erhalten. Noch in Sophokles' Antigone klingt diese skeptische Grundüberzeugung von der Endlichkeit von Leben und Wissen des Menschen an, wenn der Chor im ersten Standlied zwar »Sprache und windschnelles / Denken (phrónema) « ${ }^{5}$ preist, zugleich aber resigniert feststellt: »vor Hades allein / wird er sich kein Entrinnen schaffen $\ll{ }^{6}$ Was dem Menschen

3 Hesiod: Theogonie, V. 585.

4 Ders.: Werke und Tage, übers. u. hg v. Otto Schönberger, Stuttgart 1996, S. 11.

5 Sophokles: Antigone, übers. u. hg. v. Norbert Zink, Stuttgart 1981, V. 353354.

6 Ebd., V. 360-361. 
verwehrt bleibt, ist eine Form der Souveränität, deren Grundlage die Überschreitung des Todes wäre, der den Menschen seit der von Hesiod beschriebenen Trennung von den Göttern jedoch ein für allemal auferlegt ist. Dass eine Souveränität jenseits des Todes dennoch möglich ist, ist eine Überlegung, der sich Georges Bataille anvertraut hat. Batailles Theorie der Souveränität versucht, einen positiven Begriff der Verschwendung zu entwickeln, um im Anschluss an die Theorie der Gabe bei Marcel Mauss die Kehrseite der modernen Rationalität als eine archaische Rechtsform geltend zu machen, deren Gesetze von den Tauschbegriffen der modernen politischen Ökonomie wesentlich unterschieden sind. Die religionshistorische Reflexion auf das Opfer, das Heilige und die Erotik, die Batailles Werk bestimmt, hat Jean Baudrillard in seiner Untersuchung über den Zusammenhang zwischen dem symbolischen Tausch und dem Tod noch erweitert, indem er die Form des symbolischen Tausches in die moderne Ökonomie selbst einzutragen versucht. Vor diesem Hintergrund rekapituliert der folgende Beitrag anhand der aufeinander aufbauenden Positionen von Marcel Mauss, Georges Bataille und Jean Baudrillard zunächst die religionssoziologische Bestimmung des Tausches im Rahmen der polemischen Entgegensetzung von Archaik und Moderne der französischen Theoretiker, um in einem zweiten Schritt am Beispiel der Antigone auf die Frage nach dem Zusammenhang von Tausch und Tod zurückzukommen, die schon bei Hesiod aufgeleuchtet war und auf die Baudrillard mit dem Begriff des Symbolischen zurückzukommen versucht.

\section{Gabe und Gegengabe: Marcel Mauss}

In seinem Essai sur le don aus den Jahren 1923/24 stellt Marcel Mauss eine scheinbar simple Ausgangsfrage: »Quelle est la règle de droit et d'intérêt qui, dans les sociétés de type arrière ou archaïque, fait que le présent reçu est obligatoirement rendu? « ${ }^{7}$ Was Mauss interessiert, ist der Zusammenhang von Gabe und Gegengabe, der Zwang, auf ein Geschenk mit einem anderen zu antworten. Dass Mauss im Blick auf kanadische Indianerstämme explizit bei archaischen Gesellschaftszusammenhängen ansetzt, verdeckt dabei die eigentliche Reichweite seiner Überlegungen. Zwar geht es ihm zunächst um eine Archäologie des Sozialen, die auf die Ursprünge der menschlichen Kultur zurückführen soll: »D'une part, nous arriverons à des conclusions en quelque sorte archéologiques sur la nature des transac-

7 Marcel Mauss: "Essai sur le don. Form et raison de l'échange dans les sociétés archaïques «, in: Sociologie et anthropologie, Paris 1950, S. 143279, hier S. 148 (im Original kursiv). 
tions humaines dans les sociétés qui nous entourent ou nous ont immédiatement précédés. $\ll^{8}$ Darüber hinaus aber dient Mauss die Archäologie vergangener Kulturen als Erklärungsmodell für eine aktuelle Krisenerfahrung: »comme nous croyons avoir ici trouvé un des rocs humains sur lesquels sont bâties nos sociétés, nous pourrons en déduire quelques conclusions morales sur quelques problèmes que posent la crise de notre droit et la crise de notre économie «. ${ }^{9}$ Was Mauss vermutet, ist die Abhängigkeit der modernen Ökonomie von einer archaischen Ordnung, die es offen zu legen gilt, um nicht nur die Vergangenheit, sondern auch die Gegenwart zu begreifen. »Une partie considérable de notre morale et de notre vie ellemême stationne toujours dans cette même atmosphère du don, de l'obligation et de la liberté mêlé«, ${ }^{10}$ so lautet die überraschende Prämisse von Mauss, aus der sich nicht minder überraschende Folgerungen zur Struktur und Genese des Tauschprozesses ableiten.

Das archaische System des Tausches fasst Mauss im Begriff des potlatch, der seiner Meinung nichts anderes als ") nourrir $<$, >consommer $« "{ }^{11}$ bedeutet. Den potlatch begreift Mauss dabei als ein, wie er sich ausdrückt, totales System, das alle Seiten der Kultur umfasst: »Tous ces phénomènes sont à la fois juridiques, économiques, religieux, et même esthétiques, morphologiques, etc. ${ }^{12}$ Die grundlegende Bedeutung des potlatch für die archaischen Gesellschaften stützt Mauss durch zwei Momente: »celui de l'honneur, du prestige, du >mana que confère la richesse «, und »celui de l'obligation absolue de rendre ces dons sous peine de perdre ce >mana qu'est l'autorité elle-même ${ }^{13}{ }^{13}$ Im potlatch geht es demnach nicht im eigentlichen Sinne um den ökonomischen Erwerb, sondern vielmehr um eine Form der Souveränität, die sich in dem Verhältnis des Menschen zu den Dingen ausdrückt, die dem Tausch unterworfen bleiben. Voraussetzung der zentralen Bedeutung des potlatch im Gegensatz zu modernen Industriegesellschaften ist die animistische Vorstellung, dass den Dingen selbst eine Seele zukommt: »le lien de droit, lien par les choses, est un lien d'âmes, car la chose elle-même a une âme, est de l'âme. « ${ }^{14}$ Durch die Dinge vermittelt setzen sich die Menschen in ein agonales Verhältnis zueinander, das zugleich ihre soziale Position in der Gruppe bestimmt: »car, accepter quelque chose de quelqu'un, c'est accepter quelque chose

8 Ebd.

9 Ebd.

10 Ebd., S. 258.

11 Ebd., S. 152.

12 Ebd., S. 274, vgl. in diesem Zusammenhang auch S. 204.

13 Ebd., S. 155.

14 Ebd., S. 160. 
de son essence spirituelle, de son âme $\ll{ }^{15}$ Auf eine Gabe nicht mit einer Gegengabe antworten zu können, bedeutet im Rahmen der von Mauss diagnostizierten archaischen Tauschvorstellung daher das Eingeständnis der eigenen Unterlegenheit. Eine besondere Bedeutung kommt in diesem Zusammenhang dem Tod zu: »L'un des premiers groupes d'êtres avec lesquels les hommes ont dû contracter et qui par définition étaient là pour contracter avec eux, c'étaient avant tout les esprits des morts et les dieux. $\aleph^{16}$ Mit dem Zusammenhang von Tausch und Tod rührt Mauss am Ursprung des potlatch, zugleich aber an einer im Rahmen der Tauschprozesse schwer beschreibbaren Grenze. Denn im universellen System der Gabe ist der Tod einerseits dasjenige, was sich nicht tauschen lässt, andererseits aber auch dasjenige, was sich von allen Dingen am ehesten zum Tausch anbietet. So erfüllen die Totengaben die Form einer wechselseitigen Verpflichtung: Die Toten erhalten ihre Geschenke und versprechen dadurch, gleichsam als Gegengabe, ihre Macht, die Macht des Todes, an die Gruppe abzutreten. Der Tausch erweist sich so als eine Form der rituellen Reinigung, die Tote und Lebendige voneinander scheidet und zugleich in eine symbolische Beziehung zueinander treten lässt. So errichtet sich nach Mauss in archaischen Gesellschaften ein soziales System, das sich im Unterschied zu modernen Zivilisationsstrukturen zu wesentlichen Teilen durch das Verhältnis zu den Toten bestimmt.

Die archaische Form des Tausches, die Mauss mit dem potlatch als Grundlage des Sozialen anvisiert, deutet er daher zugleich als einen Einspruch gegen das moderne Konzept des eigennützigen Individualismus, den die kapitalistische Gesellschaftsordnung privilegiert. Mauss geht sogar so weit, eine Rückkehr zu den archaischen Ordnungen zu propagieren: »Ainsi, on peut et on doit revenir à de l'archaïque, à des éléments; on retrouvera des motifs de vie et d'action que connaissent encore des sociétés et des classes nombreuses: la joie à donner en public; le plaisir de la dépense artistique généreuse; celui de l'hospitalité et de la fête privée et publique. ${ }^{17}$ In einer überraschenden Wende fordert Mauss die Wiederkehr des Archaischen als Therapie gegen die moderne Entfremdung, die schon Marx aus der Tauschordnung des Kapitals abgeleitet hatte. Gastfreundschaft, Kunst, Generosität und Fest gelten ihm als Mittel, den modernen Individualismus zu überwinden. Mit dem Paradox einer Überschreitung der Moderne durch eine Rückwendung zur Archaik hat Mauss zugleich eine Forderung formuliert, die auf unterschiedliche Weise von Bataille und Baudrillard aufgenommen werden konnte. 


\section{Der souveräne Tausch: Georges Bataille}

»L'au-delà de l'utilité est le domaine de la souveraineté. $\aleph^{18}$ Mit dieser Unterscheidung, die die beiden Bereiche des Profanen und des Sakralen über die Begriffe des Nützlichen und des Heiligen unvermittelt gegenüberstellt, knüpft Georges Bataille an die Überlegungen von Marcel Mauss an. Wie Mauss gilt ihm das Heilige, der Ausdruck des Archaischen, als ein Bereich der Souveränität, der die Kehrseite der modernen Rationalität repräsentiert, und wie Mauss nutzt Bataille den Blick auf archaische Gesellschaftszusammenhänge zu einer grundsätzlichen Kritik an der modernen Gesellschaft:

L'homme archaïque était principalement occupé de ce qui est souverain, merveilleux, de l'au-delà de l'utile, mais c'est là justement ce qu'une conscience éclairée par le progrès des connaissances rejette dans le clair-obscur, douteux et condamnable, auquel la psychanalyse donna le nom d'inconscient. L'homme moderne ignore ou méconnaît, il tend à dénigrer ou à nier ce que l'homme achaïque a tenu pour souverain. L'homme archaïque se posait sans fin la question de la souveraineté, c'était pour lui la question première, celle qui comptait souverainement à ses yeux. ${ }^{19}$

Der kulturelle Fortschritt, so lautet die in ihrer Ausschließlichkeit nicht unproblematische These von Bataille, bedeutet in Wirklichkeit einen Verlust an Souveränität, der aus der Beschränkung der Ökonomie auf das Prinzip des Nützlichen resultiert. Mit der Souveränität spricht Bataille ein Prinzip an, das sich modernen Arbeitszusammenhängen entzieht:

Ce qui distingue la souveraineté est la consommation des richesses, en opposition au travail, à la servitude, qui produisent les richesses sans les consommer. Le souverain consomme et ne travaille pas, tandis qu'aux antipodes de la souveraineté, l'esclave, l'homme sans avoir, travaillent et réduisent leur consommation au nécessaire, aux produits sans lesquels ils ne pourraient ni subsister ni travailler. ${ }^{20}$

Bataille, der damit zugleich an die spekulative Auslegung des Verhältnisses von Herr und Knecht bei Hegel durch Alexandre Kojève anknüpft, ${ }^{21}$ bestimmt den Bereich der Souveränität als den einer politischen und religi-

18 Georges Bataille: "La souveraineté«, in: Oeuvres complètes VIII, Paris 1976, S. 243-456, hier S. 248.

19 Ebd., S. 273.

20 Ebd., S. 248.

21 Vgl. Alexandre Kojève: Introduction à la lecture de Hegel, Paris 1947, besonders S. 529-575. 
ösen Herrschaft, dessen Prinzip nicht auf Bewahrung, sondern auf Verschwendung beruht. Keine Theorie des ökonomischen Gleichgewichts interessiert Bataille, sondern die eines uneinholbaren Überschusses, den das Leben bereit hält:

Je partirai d'un fait élémentaire: l'organisme vivant, dans la situation que déterminent les jeux de l'énergie à la surface du globe, reçoit en principe plus d'énergie qu'il n'est nécessaire au maintien de la vie: l'énergie (la richesse) excédante peut être utilisée à la croissance d'un système (par exemple d'un organisme); si le système ne peut plus croître, ou si l'excédent ne peut en entier être absorbé dans sa croissance, il faut nécessairement le perdre sans profit, le dépenser, volontiers ou non, glorieusement ou sinon de façon catastrophique. ${ }^{22}$

Was Bataille in den Mittelpunkt der politischen Ökonomie stellt, ist die »énergie excédante ${ }^{23}$ als eine Form der Überfülle, die sich in der vollständigen Verausgabung erfüllt. Stabilität erreicht das soziale System durch ein Spiel von Wachstum und Verschwendung, das für ein Gleichgewicht allein dadurch sorgt, dass es sich in katastrophischen Entladungen offenbart. Bataille unterstreicht damit im Unterschied zu Mauss eher die chaotische Seite der Ökonomie, die immer wieder zu unkontrollierbaren Ereignissen wie Kriegen führt und gleichwohl eine geheime Ordnung aufrechterhält. »La vie est en son essence un excès, elle est la prodigalité de la vie. Sans limite, elle épuise ses forces et ses ressources; sans limite elle anéantit ce qu'elle a créé $\ll^{24}$, so lautet die in ihrem Pathos der Fülle auf Nietzsche zurückführende Bestimmung der Lebenskraft, die Bataille im Rahmen seiner Theorie der Souveränität vorgibt.

Wenn Bataille Leben als grenzenlose Verschwendung kennzeichnet, dann setzt er sich wie Kojève zugleich in ein Verhältnis zum Tod. Souveränität, so formuliert Bataille im Anschluss an Hegel, konstituiert sich in der Überschreitung des Todes: »Le monde souverain est le monde où la limite de la mort est supprimée. $\aleph^{25}$ Den Tod begreift Bataille daher weniger als Grenze denn als Grund der Souveränität. »Le luxe de la mort, à cet égard, est envisagé par nous de la même façon que celui de la sexualité, d'abord comme une négation de nous-mêmes, puis, en un renversement soudain, comme la vérité profonde du mouvement dont la vie est l'exposition. $\ll^{26}$ Bataille spricht vom Luxus des Todes, um dessen eigene ver-

22 Georges Bataille: "La part maudite«, in: Oeuvres complètes VII, Paris 1976, S. 17-179, hier S. 29.

23 Ebd., S. 20.

24 Ders.: "L'Ėrotisme«, in: Oeuvres complètes X, Paris 1987, S. 7-270, hier S. 88.

25 Ders.: La souveraineté, S. 270.

26 Ders.: La part maudite, S. 41. 
schwenderische Funktion hervorzuheben. Nicht nur eine Negation des menschlichen Daseins bedeute der Tod, vielmehr markiere er zugleich eine geheime Tiefendimension, die dem Leben als unaufhörliche Verschwendung seiner selbst zugrundeliege. Im Rahmen der spekulativen Verschränkung von Individuum und Gattung gelangt Bataille, nicht von ungefähr einer der Paten Foucaults, zu einer Theorie, die nicht mehr den Menschen in den Mittelpunkt stellt, sondern eine Form der Überschreitung, ${ }^{27}$ die das Leben auf die Extrembereiche von Sexualität und Tod bezieht. Der Tod erscheint so als eine Tiefendimension und zugleich als eine Bewegung der Transzendenz, die vom Opfer zutage gefördert wird und dem Leben untersteht: »C'est que généralement, dans le sacrifice ou le potlatch, dans l'action (dans l'histoire) ou la contemplation (la pensée), ce que nous cherchons est toujours cette ombre - que par définition nous ne saurions saisir - que nous n'appelons que vainement la poésie, la profondeur ou l'intimité de la passion. $\ll^{28}$ Kunst und Leidenschaft nennt Bataille als Möglichkeiten, zu der Tiefenordnung von Tod und sexueller Überschreitung Kontakt zu wahren. Zeichnet sich der kulturelle Fortschritt nach Bataille durch den Verlust der archaischen Ordnung der Souveränität aus, so bezeichnet er die Kunst geradezu als Erbe der Souveränität, ${ }^{29}$ um sie in die Tradition des Heiligen zu stellen: »La littérature se situe en fait à la suite des religions, dont elle est l'héritière. Le sacrifice est und roman, c'est un conte, illustré de manière sanglante. ${ }^{30}$ Was Bataille der Kunst, insbesondere der Literatur, zuspricht, ist die Aufrechterhaltung einer Erfahrung, die vom Verschwinden bedroht ist, weil sie mit den Gesetzen der modernen Ökonomie unvereinbar ist: »Des divers sacrifices, la poésie est le seul dont nous puissions entretenir, renouveler le feu. $\ll^{31}$ Bataille privilegiert die Literatur als Garant jener »expérience intérieure«, die im Mittelpunkt seiner nicht nur mystischen, sondern auch tendenziell mystifizierenden Darstellung von Opfer und Tausch steht. Was er mit Mauss teilt, ist die Ablehnung der politischen Ökonomie der Moderne zugunsten einer archaischen Ordnung der verschwenderischen Gabe, die ihm zugleich als Korrektur an der ausdifferenzierten Vernunft dient, die seit Hegel im Mittelpunkt des Denkens der Moderne steht. Souveränität meint in diesem Sinne eine ekstatische Erfahrung, die sich der Verdinglichung durch die instrumentelle Vernunft widersetzt ${ }^{32}$ und darin einen Freiraum begründet,

27 Vgl. Michel Foucault: "Préface à la transgression (en hommage à Georges Bataille)«, in: Dits et Écrits I. 1954-1969, Paris 1994, S. 233-250.

28 G. Bataille: La part maudite, S. 76.

29 Vgl. ders.: La souveraineté, S. 446.

30 Ders.: L'Érotisme, S. 89.

31 Ders.: »L'expérience intérieure «, in: Oeuvres complètes V, Paris 1973, S. 7289, hier S. 172.

32 Vgl. Jürgen Habermas: Der philosophische Diskurs der Moderne. Zwölf Vorlesungen, Frankfurt/Main 1988, S. 262. 
der, wie der Hinweis auf Kunst und Literatur zeigt, mit Nietzsche zugleich als ein Raum des Ästhetischen und Erbe des Sakralen zu bestimmen ist. ${ }^{33}$ So widersprüchlich Batailles Bestimmung der Souveränität auch anmuten mag: Mit der Erweiterung der Analysen von Mauss hat er den Boden bereitet, auf dem Theoretiker wie Foucault oder Baudrillard in den sechziger und siebziger Jahren des 20. Jahrhunderts weiter gearbeitet haben.

\section{Tausch und Symbol: Jean Baudrillard}

An Georges Bataille wie an Marcel Mauss kann Jean Baudrillard unmittelbar anschließen, wenn auch er vom Gegensatz von archaischer und moderner Ökonomie ausgeht: »Il n'y a plus d'échange symbolique au niveau des formations sociales modernes, plus comme forme organisatrice ${ }^{34}$, so lautet der erste Satz seiner 1976 erschienenen Studie L'échange symbolique et la mort, und mit einigem Bedauern setzt Baudrillard hinzu: »Dans le capital actuel, gigantesque machine polymorphe, le symbolique (don et contre-don, réciprocité et réversion, dépense et sacrifice) n'est plus rien $\ll^{35}$. Das Neue an Baudrillards Theorie des Tausches ist die Einführung des Begriffs des Symbolischen. Darunter versteht Baudrillard dabei zunächst jedoch nichts anderes als eine Form des Tauschaktes im Sinne von Mauss und Bataille: »Le symbolique n'est ni un concept, ni une instance ou une catégorie, ni une >structure〈, mais un acte d'échange et un rapport social qui met fin au réel, qui résout le réel, et du même coup l'opposition entre le réel et l'imaginaire. ${ }^{36}{ }^{36}$ Wenn Baudrillard sich auf die Dreierordnung des Symbolischen, Imaginären und Realen beruft, dann setzt er sich nicht nur bewusst in ein Verhältnis zu Lacan und der psychoanalytischen Tradition seit Freud. Mit der Definition des Symbolischen als einer Form des Tausches nimmt er zugleich die griechische Bedeutung des Begriffs als Zusammenführung getrennter Einheiten in den Blick. ${ }^{37}$ Das Symbolische gilt ihm dementsprechend im allgemeinen Sinne als Zeichen für die Aufhebung einer Trennung durch eine Form des

33 Das ist der Punkt, den Habermas in seiner Rekonstruktion der französischen Theoriebildung übersieht, obwohl er Baudelaire in die Ausgangsbetrachtung seiner Untersuchung miteinbezieht. Der philosophische und der ästhetische Diskurs der Moderne meinen nicht dasselbe.

34 Jean Baudrillard: L'échange symbolique et la mort, Paris 1976, S. 7.

35 Ebd., S. 61.

36 Ebd., S. 204.

37 Vgl. etwa die Bedeutung des Symbolischen für die Psychoanalyse bei Ernest Jones: Die Theorie der Symbolik und andere Aufsätze, Frankfurt/ Main, Berlin, Wien 1978, besonders S. 51. 
Tausches: »Le symbolique est ce qui met fin à ce code de la disjonction et aux termes séparés. $^{38}$

Wenn er das Symbolische auf den Bereich des Todes bezieht, knüpft Baudrillard wiederum an Mauss und Bataille an. Wie Bataille, so deutet Baudrillard den Tod zunächst als katastrophischen Einbruch in die Ordnung des Lebens: »La mort peut-être et elle seule, la réversibilité de la mort est d'un ordre supérieur à celui du code. Le désordre symbolique seul peut faire irruption dans le code. ${ }^{39}$ Katastrophisch, nicht dialektisch begreift Baudrillard den Tod, um in ihm zugleich eine soziale Demarkationslinie zu erkennen, die die Toten und die Lebendigen voneinander scheidet. Analog zu den Arbeiten von Michel Foucault, der den Ausschluss von Wahnsinn, Kriminalität und Sexualität aus der Ordnung der modernen Vernunft beschrieben hat, weist Baudrillard auf den Ausschluss des Todes aus modernen Gesellschaften hin. Baudrillard spricht in diesem Zusammenhang von einer »exclusion qui les précède toutes et leur sert de modèle, qui est à la base même de la >rationalité^ de notre culture: c'est celle des morts et de la mort. ${ }^{40}$ Die Trennung von Toten und Lebendigen verkörpert Baudrillard zufolge demnach die Matrix aller sozialen Ordnungen. Wenn er das Symbolische in diesem Kontext als eine Form der Zusammenführung des Getrennten anspricht, dann wird zugleich deutlich, dass die Funktion des Symbolischen für ihn in der Aufhebung der Trennung von Tod und Leben besteht: »La vie rendue à la mort, c'est l'opération même du symbolique. ${ }^{41}$

Wie Mauss und Bataille geht Baudrillard als Ausgangspunkt seiner Überlegungen von archaischen Gesellschaftszusammenhängen aus. In ihnen, so Baudrillard, gelte der Tod als »désordre absolu, parce que ça ne peut pas s'échanger symboliquement, et ce qui ne peut pas s'échanger symboliquement constitue un danger mortel pour le groupe. ${ }^{42}$ Dementsprechend verkörpert der Tod für Baudrillard keinen biologischen, sondern einen soziologischen Begriff. Der Tod ist dasjenige, was aus den Tauschverhältnissen ausgeschlossen bleibt und so als ständige Bedrohung über der Ordnung des Sozialen schwebt. Die Schwierigkeit für die archaischen Kulturen besteht daher darin, den Tod zum Gegenstand des Tausches zu machen. Baudrillard zufolge gelingt das durch Initiationsriten: »Il est clair que l'initiation consiste en l'instauration d'un échange là où il n'y avait que fait brut: de la mort naturelle, aléatoire et irréversible, on passe à une mort donnée et reçue, donc réversible dans l'échange social, ১soluble` dans

38 J. Baudrillard: L'échange symbolique et la mort, S. 205.

39 Ebd., S. 11.

40 Ebd., S. 195.

41 Ebd., S. 201.

42 Ebd., S. 202. 
l'échange. $\aleph^{43}$ Die Formen archaischer Initiationsriten deutet Baudrillard als Übergang von einer Ordnung des Realen und des Imaginären zu einer Ordnung des Symbolischen, wobei der durch die Initiation erreichte Fortschritt darin besteht, dasjenige, was sich dem Tausch verwehrt, im Rahmen des schon von Bataille hervorgehobenen Festes selbst zum Tauschobjekt zu machen. »La mort symbolique, celle qui n'a pas subi cette disjonction imaginaire de la vie et de la mort qui est à l'origine de la réalité de la mort, celle-là s'échange dans un rituel social de fête. $\aleph^{44} \mathrm{Im}$ Fest, so Baudrillard, gelingt die symbolische Aufhebung des Todes, die wie schon bei Mauss den Übergang vom Individuellen zum Sozialen markiert: Der symbolische Tod des Einzelnen sichert ihm seinen Platz in der Gruppe, die Gruppe selbst bewahrt sich vor der zerstörerischen Macht des Todes, die jederzeit über sie hereinzubrechen droht.

Vor diesem Hintergrund beklagt Baudrillard den Verlust des Symbolischen in der modernen politischen Ökonomie zugleich als einen Verlust des Sozialen, der zu beständiger Vereinsamung und Isolierung des Subjekts auf der einen Seite und zur vollständigen Verdrängung des Todes auf der anderen Seite führt. Wie schon Bataille, so erkennt auch Baudrillard einzig in der Literatur eine adäquate Art und Weise, sich zur symbolischen Ordnung in ein Verhältnis zu setzen, und wie Bataille begreift er die souveräne Sprache der Poesie zugleich als Subversion der modernen Ökonomie: »Dans le champ du langage aussi existe le modèle d'un échange symbolique, quelque chose comme le noyau d'une anti-économie politique, lieu d'extermination de la valeur et de la loi: c'est le langage poétique. « ${ }^{45}$ Als Wiedereinsetzung des Symbolischen auf der Ebene der Sprache markiert die Literatur für Baudrillard einen bevorzugten Wissensbereich, der an die katastrophischen Ordnungen archaischer Gesellschaften anzuknüpfen vermag. Neigt Baudrillard wie Bataille und Foucault damit mehr oder weniger zu einer Mystifizierung der poetischen Funktion der Sprache als Subversion politischer und ökonomischer Machtverhältnisse, so stellt sich zugleich die Frage, wie sich der Zusammenhang von Tausch, Opfer und Tod in der Literatur fassen lässt, die dem Bereich des Archaischen noch nahe steht und andererseits doch als Grundlage der modernen Kultur verstanden werden kann. Hatte schon Hegel in der Ästhetik von der Antigone behauptet, sie sei »das vortrefflichste, befriedigendste Kunstwerk ${ }^{46}$ aller Zeiten, so kann die griechische Tragödie im folgenden als exemplarisches Beispiel für die Frage nach dem Zusammenhang von Tausch und Tod in der literarischen Verarbeitung des Mythos dienen.

43 Ebd., S. 203.

44 Ebd., S. 226.

45 Ebd., S. 285.

46 Georg Wilhelm Friedrich Hegel: Ästhetik, Bd. 2, Berlin 1985, S. 568. 


\section{Hegel und Sophokles}

Baudrillard, der sich in einer für die französischen Theoriebildungsprozesse der siebziger Jahre charakteristischen Weise nicht nur gegen Marx, sondern auch gegen Freud wendet und allein dessen Begriff des Todestriebes als Anknüpfungspunkt für weitere Überlegungen gelten lassen will, bezieht die Form des symbolischen Tausches, die im Zentrum seiner Untersuchung steht, nicht auf die Figur des Vaters, sondern auf die der Schwester: »Ainsi la >fonction symbolique dans les sociétés primitives ne s'articule pas sur la loi du Père et le principe de réalité psychique individuel, mais d'emblée sur un principe collectif, sur le mouvement collectif des échanges. ${ }^{47}$ Mit dem Gesetz des Vaters lehnt Baudrillard wie Deleuze/ Guattari in ihrem Anti-Oedipe Freuds Begriff des Ödipalen ab, um das Blickfeld auf Geschwisterverhältnisse zu verschieben: »Au principe de l'Oedipe, qui correspond à l'aspect négatif de l'interdit de l'inceste (interdit sur la mère imposé par le père) s'oppose, dans le sens positif, un principe d'échange des sœurs par les frères - c'est la sœur, pas la mère, qui est au centre du dispositif, et c'est au niveau des frères et sœurs que s'organise tout le jeu social des échanges. $\aleph^{48}$ Mit der These, die Schwester stünde im Mittelpunkt der symbolischen Tauschprozesse, führt Baudrillards antifreudianische Volte im vergleichenden Blick auf die griechische Tragödie nicht auf den Ödipus, sondern auf die Antigone zurück.

Die Bedeutung des Geschwisterverhältnisses in der Antigone hat schon Hegel angesprochen. Hegels Interpretation der Antigone in der Phänomenologie des Geistes steht wie bereits die der Orestie im Naturrechtsaufsatz im Kontext des Opfers. In der Orestie erkennt Hegel eine durch das Opfer geleistete Versöhnung, die »eben in der Erkenntnis der Notwendigkeit und in dem Rechte besteht, welches die Sittlichkeit ihrer unorganischen Natur und den unterirdischen Mächten gibt, indem sie ihnen einen Teil ihrer selbst überläßt und opfert « ${ }^{49}$, wobei Hegel das Opfer in der Tradition der aristotelischen Lehre von der katharsis zugleich als eine Reinigung begreift, derzufolge »das Lebendige aber, indem es das, was es als einen Teil seiner selbst weiß, in dasselbe legt und dem Tode opfert, dessen Recht zugleich anerkannt und zugleich sich davon gereinigt hat. $\aleph^{50}$ Was die Tragödie demnach leistet, ist eine Anerkennung und

47 J. Baudrillard: L'échange symbolique et la mort, S. 209.

48 Ebd.

49 Georg Wilhelm Friedrich Hegel: „Über die wissenschaftliche Behandlung des Naturrechts, seine Stelle in der praktischen Philosophie und sein Verhältnis zu den positiven Rechtswissenschaften «, in: ders., Werke 2: Jenaer Schriften 1801-1807, Frankfurt/Main 1986, S. 434-530, hier S. 494. Ebd., S. 495. 
darin eine Reinigung von der Macht des Todes, deren Recht in der Orestie von den Erinnyen vertreten wird.

Indem Hegel in der Phänomenologie des Geistes nicht mehr die Orestie, sondern die Antigone zum Vorbild nimmt, verlagert er den Konflikt, der sich in der Orestie zwischen Orest und den Erinnyen entspannt, in einer nicht unproblematischen Weise auf den Streit von Kreon und Antigone. Hegel hat in diesem Zusammenhang mit drei fundamentalen Problemen zu kämpfen, die zugleich seinen eigenen Begriff der Dialektik betreffen: ${ }^{51}$ Mit der Antigone und der Orestie stehen sich eine Einzeltragödie und eine Trilogie, weiblicher und männlicher Protagonist und schließlich Katastrophe und Versöhnung gegenüber. Das erste Problem sucht Hegel zu lösen, indem er die prozessuale Struktur der Trilogie, die für den eigenen Begriff der Dialektik von entscheidender Bedeutung ist, durch die Konstruktion einer neuen Trilogie um den Ödipus auf Kolonos, die Sieben gegen Theben und der Antigone zu bewahren sucht. Ausgangspunkt des Streites sei Ödipus' Fluch über seine beiden Söhne, der Kampf um Theben gilt ihm entsprechend als Entzweiung des Gesetzes in die beiden Seiten der feindlichen Brüder, wobei die Antigone schließlich den Streit beendet, indem sie das gleiche Recht der beiden Brüder durchsetzt. Problematisch bleibt in diesem Zusammenhang aber nicht nur die von Hegel nur verzerrt dargestellte Geschlechterproblematik, die in der Antigone aufscheint, wenn sich in Antigone und Kreon in ganz anderer Weise als in der Orestie die Macht des Weiblichen und die des Männlichen gegenüberstehen. Fragwürdig ist insbesondere die dialektische Struktur der Versöhnung, die Hegel unmittelbar aus der Orestie ableiten konnte, der der katastrophische Schluss der Antigone aber widerspricht. Vor diesem Hintergrund bietet es sich an, in ähnlicher Weise wie Hölderlin die Tragödie gegen Hegels Interpretation von ihrem Schluss her zu beleuchten.

\section{Mythos und Distanz: Antigone}

In seinem Vergleich des Ödipus und der Antigone hatte bereits Hölderlin darauf hingewiesen, dass die Zäsur der Tragödie im letzteren Fall mehr gegen das Ende hin neigt. ${ }^{52}$ Hölderlin trägt damit der extremen Beschleunigung Rechnung, die den Schluss der Tragödie bestimmt: Nach dem Auftritt des Sehers Teiresias eilt der verwirrte Kreon zu der Kammer, in der Antigone lebendig begraben werden soll. Neben der erhängten Königs-

51 Vgl. Peter Szondi: »Versuch über das Tragische«, in: ders., Schriften I, Frankfurt/Main 1978, S. 149-260, hier S. 167.

52 Vgl. Friedrich Hölderlin: »Anmerkungen zur Antigone«, in: Günter Mieth (Hg.), Sämtliche Werke und Briefe, Bd. 2, München, Wien 1970, S. 451458, hier S. 451. 
tochter findet er dort seinen Sohn, der sich beim Anblick des Vaters tötet. Als er wieder in die Stadt kommt, hat sich seine Frau Eurydike aufgehängt. Von einer wie auch immer gearteten Versöhnung, die Hegel der Tragödie unterstellt, ist in der Antigone jedenfalls nichts zu spüren, und auch der abschließende Hinweis des Chores auf die phronésis, die philosophische Tugend der Besonnenheit, die als einziges dem Menschen Glück zu schenken vermag, irritiert den Zuschauer mehr, als dass sie ihn zu beruhigen vermöchte.

Den Beginn des katastrophischen Endes und damit die Zäsur markiert, wie wiederum bereits Hölderlin betont hat, der Auftritt von Teiresias. Dem Seher geht es seiner priesterlichen Funktion gemäß um die Frage des Opfers, und wie schon im Fall der pestverseuchten Stadt, als deren Urheber Ödipus auszumachen war, geht es beim Auftritt des Teiresias zunächst um die Feststellung einer fundamentalen Störung des Opferprozesses, als deren Schuldiger nun Kreon erscheint: »Denn die Altäre und die hochheiligen Opferstätten sind uns / voll vom Fraß der Vögel und Hunde / von dem unselig gefallenen Ödipussohn. / Und so nehmen die Götter denn nicht mehr die Opfergebete an / von uns und auch der Lenden Dampf, / und kein Vogel läßt Geschrei ertönen als frohes Zeichen, / weil sie gegessen haben vom Fett eines Ermordeten. ${ }^{53}$ Teiresias macht mit seinen Worten auf das zentrale Problem des Stückes aufmerksam: auf die Frage nach der Bestattung des gefallenen Polyneikes, die Kreon verboten hatte und dessen geschändeter Leichnam vor den Mauern der Stadt verrottet. Dass die Vögel und Hunde von dem Fleisch des Ermordeten gefressen haben, deutet auf eine Störung innerhalb des Verhältnisses von Menschen und Göttern hin, die darauf zurückgeht, dass der Nichtbestattete die Stadt mit seinem Körper befleckt.

Vor diesem Hintergrund wirft die Rede des Teiresias zugleich ein bezeichnendes Licht auf die Tat Antigones. Nichts anderes, als die Stadt vor der Befleckung durch den Toten zu retten, war ja ihr Ziel. Die Bestattung, die sie ausdrücklich gegen das Verbot Kreons vornimmt, ist daher vor allem als ein symbolischer Akt zu sehen. Das wird in der Schilderung der unerhörten Tat durch den Wächter deutlich: »Die Leiche hat gerade einer / bestattet und ist weg, auf den Leib streute er / trockenen Staub und brachte Spenden, wie es sich gehört. ${ }^{54}$ Wie es sich gehört: Antigone vollzieht an ihrem Bruder einen Totenritus, der nicht nur dessen Wohl nach dem Tode gilt, sondern zugleich dem der Stadt, als deren Sohn er geboren wurde. Von einer eigentlichen Bestattung kann dabei zugleich keine Rede sein. Alles, was Antigone tut, ist, ihren Bruder mit einer dünnen Schicht Staub zu bedecken: »Denn die Leiche war nicht etwa weggeschafft, nicht begra-

53 Sophokles: Antigone, V. 1016-1022.

54 Ebd., V. 245-247. 
ben, / sondern auf ihr lag dünner Staub wie von einem, der einen Frevel meiden will. ${ }^{55}$ Der Wächter unterstreicht in seiner zu lakonischer Kürze neigenden Darstellung noch die letztlich symbolische Bedeutung der Beerdigung: Der harte Boden erlaubt es nicht, ein Grab zu schaufeln, dennoch muss der Tote rituell mit der Erde vermählt werden.

Gerade die rituelle Bedeutung der Bestattung verkennt Kreon jedoch. Er vermutet Bestechung: »Kein ärgerer Brauch als die Liebe zum Geld / erwuchs den Menschen ${ }^{56}$, so lautet sein Diktum. Darin wird deutlich, dass Antigone und Kreon im Rahmen der Tragödie von einer ganz unterschiedlichen Auffassung des Ökonomischen ausgehen: Kreon kennt nur die Zirkulation von Geld, Antigone geht es um die Zirkulation von Leben und Tod. Daher benutzt sie, um ihr eigenes Schicksal vorzustellen, in einer schrecklichen Doppeldeutigkeit das gleiche Wort wie Kreon, Gewinn (kérdos), um die Notwendigkeit ihres eigenen Todes zu betonen: »Wenn vor der Zeit / ich sterben werde, nenne ich es nur Gewinn. $\ll^{57}$

Der Schluss der Tragödie wiederholt den Konflikt zwischen einer Ökonomie, die auf das Geld gerichtet ist, und einer, die sich aus dem Tod ableitet, wenn Kreon Teiresias analog zu dem Wächter vorwirft, aus Liebe zum Geld zu handeln: »Sucht euren Gewinn, erhandelt euch von Sardes / das Hellgold und, wenn ihr wollt, das indische / Gold; im Grabe werdet ihr jenen nicht bergen. ${ }^{58}$ Erst diese dramatische Verkennung der Sachlage lässt Teiresias eine Drohung aussprechen, die Kreon erschüttert und seinen Sinn wandelt:

So wisse denn, daß du nicht mehr viele / rasch rollende Kreise der Sonne erleben wirst, / innerhalb deren du selbst von deinen Blutsverwandten einen / Toten den Toten zum Entgelt dagegen geben mußt, / dafür, daß du von den Oberen hinabstürztest / ein Leben und ihm ehrlos ein Grab als Zuhause gabst / und daß du hier festhältst der unterirdischen Götter / unteilhaftig, unbestattet, ungeweiht einen Toten. ${ }^{59}$

Den Toten den Toten zum Entgelt: Was Teiresias anspricht, ist eine Form des Tausches, die sich unterhalb der Ebene des Geldes bewegt, weil sie allein natürliche Äquivalenzen kennt. Die strukturelle Aufeinanderbezogenheit von Antigone und Kreon, die Hegel zu seiner umstrittenen These von der Gleichberechtigung der sittlichen Mächte geführt hat, beruht darauf, dass Antigone zwar Kreons Gesetz erleidet, Kreon aber umgekehrt das Antigones. Der Tausch sieht vor, dass er, der für den Tod Antigones und

55 Ebd., V. $255 f$.

56 Ebd., V. $295 f$.

57 Ebd., V. $461 f$.

58 Ebd., V. 1037-1039.

59 Ebd., V. 1064-1071. 
die Nichtbestattung von Polyneikes verantwortlich ist, den Toten aus der eigenen Familie nehmen muss. Auf Antigones Tod antwortet der Hämons, und Kreon verliert sein einziges Kind und damit den Anspruch auf die Herrschaftsnachfolge. Insofern erfüllt das katastrophische Ende einen Tauschprozess, der sich zwischen den Toten errichtet.

Das Ziel des symbolischen Tausches liegt dabei, durchaus im Sinne Baudrillards, darin, die Stadt von der Befleckung durch den Tod zu reinigen. Das verdeutlicht das fünfte Standlied des Chores, das in unterschiedlichen Namen den Gott Dionysos als Beschützer der Stadt anruft: »Die Stadt ehrst du / als höchste von allen, / zusammen mit der blitzgetroffenen Mutter; / auch jetzt, wo von gewaltiger / Krankheit die Stadt mit dem gesamten Volk erfaßt wird, / komm mit reinigendem Fuß über des Parnaß / Hügel oder die tosende Furt. $"{ }^{60}$ Was der Chor bezweckt, ist die Epiphanie des Gottes, der mit all seiner Macht, »zusammen mit deinen Dienerinnen, / die dich im Rausch die ganze Nacht hindurch / betanzen «, ${ }^{61}$ die Stadt vom Frevel befreien soll. »Dionysos, dem zu Ehren sie sich wie Verrückte und Rasende benehmen, ist ja derselbe wie Hades «, ${ }^{62}$ so lautet ein dunkles Wort von Heraklit, das sich im fünften Standlied zu bestätigen scheint. In der Antigone tritt Dionysos als Hades auf, der die Toten zu sich holt. Insofern verkörpert die Anrufung des Dionysos all jene Momente, die Bataille später mit dem Begriff der Verschwendung verbinden wird: In einer Form der Ekstase, einem Fest in der Gestalt des nächtlichen Tanzes, hält der Gott Einzug in die Stadt, um nach dem Gesetz von Gabe und Gegengabe nicht nur Antigone, sondern auch Hämon zu sich zu holen. Die Epiphanie des Gottes fördert damit die von Kreon verleugnete Kehrseite einer Ökonomie hervor, die auch der Herrscher der Stadt nicht mehr zu kontrollieren weiß, sondern von der er selbst beherrscht wird. So gleicht Kreon zum Schluss der Tragödie selbst einem »lebendigen / Toten «, ${ }^{63}$ einem Toten allerdings, dem es im Unterschied zu Antigone nicht vergönnt ist, in den Hades zu steigen, weil der Tauschprozess bereits abgeschlossen ist: »Es soll kommen, soll kommen / meiner Geschicke schönstes, soll erscheinen, / das den letzten Tag mir bringt / das allerletzte. Es komme doch, / damit ich nicht mehr den nächsten Tag sehen muß.« ${ }^{64}$

Der Tod kommt aber nicht. An Kreon und Ismene, Antigones Schwester, die sich der Tat verweigerte, ist er vorbeigegangen. Damit bestätigt der Schluss der Antigone, was sich schon bei Hesiod angedeutet hatte: Als Kin-

60 Ebd., V. 1137-1145.

61 Ebd., V. 1150-1152.

62 »Heraklit«, in: Die Vorsokratiker I. Mileser, Pythagoreer, Xenophanes, Heraklit, Parmenides. Auswahl der Fragmente, übers. u. erl. v. Jaap Mansfeld, Stuttgart 1983, S. 231-283, hier S. 253.

63 Sophokles: Antigone, V. 1167.

64 Ebd., S. 1328-1332. 
der des Betrügers und Feuerräubers Prometheus stehen die Menschen nicht auf eigenen Füßen; was ihnen verwehrt bleibt, ist sowohl das Wissen um das Gute als auch die Einsicht in das Ende des eigenen Daseins, die von anderen Mächten abhängt als den eigenen. Der Tauschprozess, den die Antigone zwischen Lebendigen und Toten vorführt, führt so auf die dem Wissen verborgene Sphäre der unterirdischen Macht des Todes zurück. Besonnenheit, die der Chor zum Schluss wider das eigene Unwissen anpreist, besteht anscheinend darin, die Bezogenheit des Menschen auf die ihm verschlossene Macht der Endlichkeit anzuerkennen. Nichts anderes forderte auch Bataille in seiner Beschreibung der Souveränität als einer Form der Gabe, die auf der Verschwendung basiert und zu den letztlich mystisch fundierten Erfahrungen von Sexualität und Tod zurückführt. »Aber nicht das Leben, das sich vor dem Tode scheut und von der Verwüstung rein bewahrt, sondern das ihn erträgt und in ihm sich erhält, ist das Leben des Geistes « ${ }^{65}$, hatte Hegel in der Phänomenologie des Geistes formuliert und damit auch den Geist einem Tauschprozess von Leben und Tod unterworfen.

Ob Exzess im Sinne Batailles oder Bewahrung im Sinne Hegels: Was die griechische Tragödie vorführt, ist eine grundsätzlich andere Bewegung als die von Hegel und Bataille diagnostizierte Integration oder Überschreitung des Todes. Die Dezentriertheit des menschlichen Subjekts, die postmoderne Theorien im Rahmen der Kritik der modernen politischen Ökonomie formulieren, ruht bei Hesiod wie bei Sophokles auf der Präsenz einer mythischen Gewalt, die weder von der Vernunft kontrolliert noch durch eine wie auch immer geartete Erfahrung eingeholt werden kann, weil sie, wie Hölderlin festgehalten hat, »den Menschen seiner Lebenssphäre, dem Mittelpunkt seines innern Lebens in eine andere Welt entrückt und in die exzentrische Sphäre der Toten reißt. « ${ }^{66}$ Im Blick auf Antigone hält Hölderlin im Unterschied zu Hegel daher fest, dass sie sich nicht, wie das Leben des Geistes, von der Verwüstung rein bewahrt, sondern selbst »ein wüst gewordenes Land ${ }^{67}$ verkörpert, einen Körper mithin, in dem »der Gott in der Gestalt des Todes gegenwärtig ist $\ll .{ }^{68}$ Nicht mit dem toten Bruder vermählt sich Antigone, wie noch Judith Butler in ihrer kritischen Auseinandersetzung mit Hegel und Lacan suggeriert, ${ }^{69}$ sondern, in der

65 Georg Wilhelm Friedrich Hegel: Werke, Bd. 3: Phänomenologie des Geistes, Frankfurt/Main 1986, S. 36.

66 Friedrich Hölderlin: »Anmerkungen zum Ödipus«, in: G. Mieth (Hg.), Sämtliche Werke und Briefe, Bd. 2, S. 389-396, hier S. 391.

67 Ders.: Anmerkungen zur Antigone, S. 454.

68 Ebd., S. 455.

69 »Der Tod figuriert hier also als eine Art Hochzeit mit denen aus ihrer Familie, die bereits tot sind, und so wird die Todesartigkeit jener Liebe betont, für die es in der Kultur keinen haltbaren und lebbaren Platz gibt. « (Judith Butler: Antigones Verlangen. Verwandtschaft zwischen Leben und Tod, Frankfurt/Main 2001, S. 47.) Butler, die die Antigone personenzen- 
Form des »Antitheos $«,{ }^{70}$ mit dem Gott, der sich dem Menschen nur in der Gestalt des Todes zu öffnen vermag. Indem Antigones Leib gewaltsam in die exzentrische Sphäre des Todes gerissen wird, markiert sie für Hölderlin in der allegorischen Bedeutung eines von göttlicher Naturgewalt zerstörten weiblichen Körpers wie das von ihr selbst zum Vergleich zitierte Beispiel der Niobe jedoch zugleich eine Form des Opfers, die aus dem symbolischen Tauschprozess herausfällt. Wenn Antigone sich in ihrem letzten Auftritt im Klagelied als Vermählte des Todes bezeichnet - »ich werde Acheron angetraut ${ }^{71}{ }^{71}$, dann drückt sich darin keine Erotisierung des Todes aus, sondern, in einer mit Hölderlin als tendenziell lyrisch zu bezeichnenden Weise, eine Abschiedsformel, die bis zuletzt dem Tod zu widerstehen sucht und ihn doch gegen den eigenen Willen zulassen muss. Was Sophokles in der Antigone festhält, ist daher nicht allein der gewaltsame Vollzug des Opfers, wie ihn Bataille für die Moderne fordern wird, sondern zugleich die Trauer, die sich vom Mythos distanziert. Als Inkarnation der Trauer, als die Antigone dem Zuschauer in ihrem letzten Auftritt vor Augen steht, scheidet sich der Text, den Hölderlin daher als Ausdruck einer poetologischen Zeitenwende verstehen konnte, von der archaischen Gewalt, deren unbeherrschbare Macht Sophokles beschreibt, ohne sich ihr doch zu verschreiben. Erst in dieser Distanz etabliert sich die Literatur der Moderne, auf die Bataille und Baudrillard rekurrieren, als Abschied vom Mythos und damit nicht als Wiedereinsetzung, sondern als Suspension des Opfers jenseits archaischer Tauschprozesse.

\section{Literaturverzeichnis}

Bataille, Georges: »L'expérience intérieure«, in: Oeuvres complètes V, Paris 1976, S. 7-289.

Bataille, Georges: »La part maudite«, in: Oeuvres complètes VII, Paris 1976, S. 17-179.

Bataille, Georges: »La souveraineté«, in: Oeuvres complètes VIII, Paris 1976, S. 243-456.

Bataille, Georges: »L'Érotisme«, in: Oeuvres complètes X, Paris 1976, S. 7-270.

Baudrillard, Jean: L'échange symbolique et la mort, Paris 1976.

Butler, Judith: Antigones Verlangen. Verwandtschaft zwischen Leben und Tod, Frankfurt/Main 2001.

triert als »Allegorie für die Krise der Verwandtschaft « (ebd., S. 48) liest, bleibt damit in ihrer Kritik an Hegel und Lacan der psychoanalytischen Lesart der Tragödie gegen die eigene Intention tief verpflichtet.

70 F. Hölderlin: Anmerkungen zur Antigone, S. 455.

71 Sophokles: Antigone, V. 816. 
Foucault, Michel: »Préface à la transgression (en hommage à Georges Bataille)«, in: Dits et Écrits I. 1954-1969, Paris 1994, S. 233-250.

Habermas, Jürgen: Der philosophische Diskurs der Moderne. Zwölf Vorlesungen, Frankfurt/Main 1988.

Hegel, Georg Wilhelm Friedrich: Ästhetik, Bd. 2, Berlin 1985.

Hegel, Georg Wilhelm Friedrich: „Über die wissenschaftliche Behandlung des Naturrechts, seine Stelle in der praktischen Philosophie und sein Verhältnis zu den positiven Rechtswissenschaften «, in: ders., Werke 2: Jenaer Schriften 1801-1807, Frankfurt/Main 1986, S. 434-530.

Hegel, Georg Wilhelm Friedrich: Werke, Bd. 3: Phänomenologie des Geistes, Frankfurt/Main 1986.

»Heraklit «, in: Die Vorsokratiker I. Mileser, Pythagoreer, Xenophanes, Heraklit, Parmenides. Auswahl der Fragmente, übers. u. erl. v. Jaap Mansfeld, Stuttgart 1983, S. 231-283.

Hesiod: Theogonie, hg., übers. u. erl. v. Karl Albert, 3. Aufl., Sankt-Augustin, Richarz 1985.

Hesiod: Werke und Tage, übers. u. hg. v. Otto Schönberger, Stuttgart 1996.

Hölderlin, Friedrich: »Anmerkungen zum Ödipus und Anmerkungen zur Antigone«, in: Günter Mieth (Hg.), Sämtliche Werke und Briefe, Bd. 2, München, Wien 1970, S. 389-396 und S. 451-458.

Jones, Ernest: Die Theorie der Symbolik und andere Aufsätze, Frankfurt/ Main, Berlin, Wien 1978.

Kojève, Alexandre: Introduction à la lecture de Hegel, Paris 1947.

Mauss, Marcel: »Essai sur le don. Form et raison de l'échange dans les sociétés archaïques «. in: Sociologie et anthropologie, Paris 1950, S. 143-279.

Sophokles: Antigone, übers. u. hg. v. Norbert Zink, Stuttgart 1981.

Szondi, Peter: »Versuch über das Tragische«, in: ders., Schriften I, Frankfurt/ Main 1978, S. 149-260.

Zoepffel, Renate: »Die List bei den Griechen«, in: Harro von Senger (Hg.), Die List, Frankfurt/Main 1999, S. 111-133. 
